\title{
Reducing Constraints on Quantum Computer Design by Encoded Selective Recoupling
}

\author{
D.A. Lidar and L.-A. Wu \\ Chemical Physics Theory Group, University of Toronto, 80 St. George Str., Toronto, Ontario M5S 3H6, Canada
}

\begin{abstract}
The requirement of performing both single-qubit and two-qubit operations in the implementation of universal quantum logic often leads to very demanding constraints on quantum computer design. We show here how to eliminate the need for single-qubit operations in a large subset of quantum computer proposals: those governed by isotropic and $X X Z, X Y$-type anisotropic exchange interactions. Our method employs an encoding of one logical qubit into two physical qubits, while logic operations are performed using an analogue of the NMR selective recoupling method.
\end{abstract}

Most proposals for quantum computer (QC) design rely on the execution of both single-qubit and two-qubit operations. Typically these two types of operations involve rather different manipulations and constraints. This often leads to serious technical difficulties, a problem which has been recognized and addressed in the context of QC proposals with isotropic Heisenberg spinexchange interactions [1,2], through the use of quantum codes [3 9]. It is important to note that isotropic exchange is an idealization which in reality is likely to be perturbed due to surface and interface effects, as well as spin-orbit coupling [10]. We focus here primarily on QC proposals that are governed by anisotropic exchange interactions 11 15 (the $X X Z$ and $X Y$ models, defined below). These systems either share some of the difficulties in implementing single qubit gates exhibited by Heisenberg systems 1. 2] (e.g., in the case of the quantum Hall proposal [11, extreme $g$-factor engineering, highly localized and inhomogeneous magnetic fields, and heating due to the high-intensity RF field needed for single-spin operations), or have other problems resulting from the need to implement both single and two qubit gates. E.g., in the quantum dots in cavities proposal [12] elimination of the single-qubit operations would halve the number of lasers, significantly simplifying the experimental setup. There is therefore a compelling motivation to re-examine the need for single-qubit operations in the execution of quantum logic. Here we show how the complications associated with single-qubit operations can be avoided through the use of NMR-like recoupling methods 16] applied to an encoding of two physical qubits into one logical qubit, which we developed in 17]. Our method allows universal quantum logic to be attained through switching on/off exchange interactions only, without requiring external single-qubit operations. The method requires a modest overhead in the number of physical qubits and gate operations, but this seems like a fair price to pay in return for the reduction in complexity of experimental setup. Through the use of recoupling we are able to unify the treatment of both isotropic and anisotropic exchange. Similar to [8,9] but using less stringent meth- ods, we show how to reduce the overhead in the encoding proposed earlier for the isotropic case [3 6] from three physical qubits per logical (encoded) qubit to two, under the assumption that the single-particle spectrum is nondegenerate. In the $X X Z$ case our implementation of the controlled-phase (CPHASE) gate uses as few as 4 interactions (in parallel mode on qubits arranged in 1D). The efficient encoding, and the small overhead in number of gate applications we report here, suggests that the hurdle of single-qubit operations may be overcome in forthcoming experiments implementing elementary quantum logic in isotropic or anisotropic condensed or gas phase systems [1, 2, 11, 15].

Exchange Hamiltonians.- Using spin notation, the exchange interaction quite generally 18 has the form $H_{\mathrm{ex}}=\sum_{\alpha=x, y, z} \sum_{i<j} J_{i j}^{\alpha} \sigma_{i}^{\alpha} \sigma_{j}^{\alpha}$, where $\sigma_{i}^{\alpha}$ are the Pauli matrices, and the summation is over all qubit pairs $i, j$. Tunability of the exchange constants $J_{i j}^{\alpha}$ is at the heart of all solid-state proposals, and has been studied in detail, e.g., in [1]. The isotropic (Heisenberg) case corresponds to $J_{i j}^{\alpha} \equiv J_{i j}$. The $X Y$ model is the case $J_{i j}^{x}=J_{i j}^{y}$, $J_{i j}^{z}=0$. Examples of $\mathrm{QC}$ proposals that fall into this category are: the quantum Hall proposal [11], quantum dots 12,13] and atoms in cavities 114]. The $X X Z$ model is the case $J_{i j}^{x}= \pm J_{i j}^{y} \neq J_{i j}^{z}$. [We refer to $+(-)$ as the axially symmetric (antisymmetric) case.] When surface and interface effects are taken into account the $X Y$ examples, as well as the Heisenberg examples [1] 2], are better described by the axially symmetric $X X Z$ model. Additional sources of non-zero $J_{i j}^{z}$ in the $X Y$-examples can be second-order effects (e.g., virtual cavity-photon generation without spin-flips in 12]). A natural $X X Z$ example is that of electrons on helium [15]. All these QC proposals were originally supplemented with external single qubit operations, which can be written as $F=\sum_{i} f_{i}^{x} \sigma_{i}^{x}+f_{i}^{y} \sigma_{i}^{y}$. As argued above, these operations almost invariably lead to various (system-specific) difficulties, so we will not assume that they are available. In general one must also consider the free Hamiltonian $H_{0}=\sum_{i} \frac{1}{2} \varepsilon_{i} \sigma_{i}^{z}$, where $\varepsilon_{i}$ is the single-particle spectrum. In general this spectrum will be non-degenerate, e.g., due 
to different local $g$-factors [1,2]. Which of the internal parameters $\left\{J_{i j}^{\alpha}, \varepsilon_{i}\right\}$ are controllable is a system-specific question, as summarized in Table 1. We now proceed to show how to perform (encoded) universal quantum computation while respecting the constraints imposed upon controllability of $\left\{J_{i j}^{\alpha}, \varepsilon_{i}\right\}$ by the various systems.

Encoding and operations. - First, we rewrite the general exchange Hamiltonian in a form which emphasizes axial symmetry:

$$
H_{\mathrm{ex}}=\sum_{i<j} J_{i j}^{-} R_{i j}^{x}+J_{i j}^{+} T_{i j}^{x}+J_{i j}^{z} \sigma_{i}^{z} \sigma_{j}^{z},
$$

where

$$
T_{i j}^{x}=\frac{1}{2}\left(\sigma_{i}^{x} \sigma_{j}^{x}+\sigma_{i}^{y} \sigma_{j}^{y}\right), R_{i j}^{x}=\frac{1}{2}\left(\sigma_{i}^{x} \sigma_{j}^{x}-\sigma_{i}^{y} \sigma_{j}^{y}\right),
$$

and $J_{i j}^{ \pm}=J_{i j}^{x} \pm J_{i j}^{y}$. Thus the axially symmetric (antisymmetric) case corresponds to $J_{i j}^{-}=0\left(J_{i j}^{+}=0\right)$. Note that $T_{i j}^{x}$ can also be written as $\sigma_{i}^{+} \sigma_{j}^{-}+\sigma_{i}^{-} \sigma_{j}^{+}$[where $\left.\sigma_{i}^{ \pm}=\left(\sigma_{i}^{x} \pm i \sigma_{i}^{y}\right) / 2\right]$, i.e., resonant energy transfer between qubit pairs. An important example of this is the Förster process, whereby through a Coulomb interaction an exciton hops between neighboring quantum dots that are sufficiently close. This has been used to show that a variety of quantum information processing tasks, such as the preparation of entangled states of excitons, can be performed in coupled quantum dots [13]. Our results apply to this scenario as well. In the axially symmetric case our code is simply: $\left|0_{L}\right\rangle_{m}=|\uparrow\rangle_{2 m-1} \otimes|\downarrow\rangle_{2 m}$ and $\left|1_{L}\right\rangle_{m}=|\downarrow\rangle_{2 m-1} \otimes|\uparrow\rangle_{2 m}$ for the $m^{\text {th }}$ encoded qubit, $m=1, \ldots, N / 2$. In the axially antisymmetric case: $\left|0_{L}\right\rangle=|\uparrow \uparrow\rangle$ and $\left|1_{L}\right\rangle=|\downarrow \downarrow\rangle$ (in simplified notation). Thus, logical qubits correspond to pairs of nearest neighbor physical qubits (e.g., spins). Preparation and measurement of these states was discussed in Ref. [17]. Briefly, preparation relies on relaxation to the ground state of the Hamiltonian $T_{i j}^{x}\left(R_{i j}^{x}\right)$ in the axially symmetric (antisymmetric) case, while measurement (which can also be used for preparation) employs an analogue of Kane's a.c. capacitance scheme [2]. It is important to note that none of the terms in $H_{\mathrm{ex}}$ is capable of flipping spins $i, j$ separately. Therefore the axially symmetric and antisymmetric subspaces are decoupled. This means that we can independently operate on the corresponding subspaces. Our discussion below is carried out in tandem for these two cases. With the single number $m$ serving to label our encoded qubits, it is advantageous to compactify our notation further. Let $J_{m}^{\alpha} \equiv J_{2 m-1,2 m}^{\alpha}(\alpha=z, \pm)$, and $\epsilon_{m}^{ \pm} \equiv\left(\varepsilon_{2 m-1} \pm \varepsilon_{2 m}\right) / 2$.

Let us now introduce operators which implement rotations on the encoded qubits. Let $T_{m}^{x} \equiv T_{2 m-1,2 m}^{x}$ and $T_{m}^{z}=\frac{1}{2}\left(\sigma_{2 m-1}^{z}-\sigma_{2 m}^{z}\right) ; R_{m}^{x} \equiv R_{2 m-1,2 m}^{x}$ and $R_{m}^{z}=$ $\frac{1}{2}\left(\sigma_{2 m-1}^{z}+\sigma_{2 m}^{z}\right)$, where $T_{2 m-1,2 m}^{x}$ and $R_{2 m-1,2 m}^{x}$ were defined in Eq. (2). As we showed in [17, $T_{m}^{x}\left(R_{m}^{x}\right)$ acts as the Pauli $\sigma^{x}$ matrix on the axially symmetric (antisymmetric) $m^{\text {th }}$ encoded qubit. Similarly, $T_{m}^{z}\left(R_{m}^{z}\right)$ acts as $\sigma^{z}$. Therefore pairs of these operators each generate an "encoded $S U(2)$ " group on the logical qubits, i.e., the group of all single-encoded-qubit operations. Moreover, $\left[T_{m}^{\alpha}, R_{m}^{\beta}\right]=0$ (in agreement with the decoupling of the symmetric and antisymmetric subspaces), so that these single-encoded-qubit operations can be implemented in classical parallelism. Let us now momentarily assume that we have independent control over all parameters $\left(\epsilon_{m}^{ \pm}, J_{i j}^{ \pm}, J_{i j}^{z}\right)$. Below we will relax this constraint by using selective recoupling. In order to implement singleencoded-qubit operations, we turn on the interactions $J_{m}^{ \pm}$and the energy sums and differences $\epsilon_{m}^{ \pm}$, while leaving off the interactions between spins belonging to different encoded qubits (i.e., $J_{2 m, 2 m+1}^{\alpha}=0$ ), as well as leaving all $J_{i j}^{z}$ off. We can then rewrite the total Hamiltonian $H=H_{0}+H_{\text {ex }}$ as:

$$
H=\sum_{m=1}^{N / 2}\left(\epsilon_{m}^{-} T_{m}^{z}+J_{m}^{+} T_{m}^{x}\right)+\left(\epsilon_{m}^{+} R_{m}^{z}+J_{m}^{-} R_{m}^{x}\right)
$$

while omitting a constant term. Written in this form, it is clear that by selectively turning on/off the parameters $\epsilon_{m}^{-}, J_{m}^{+}\left(\epsilon_{m}^{+}, J_{m}^{-}\right)$for the axially symmetric (antisymmetric) qubit, one can implement all single-encoded-qubit operations, by using Euler angle rotations to generate the "encoded $S U(2)$ " group. Moreover, $H$ is expressed as a sum over terms acting on different encoded qubits, so that all $N / 2$ encoded qubits (of a given symmetry) can be operated on independently. In other words, the encoded Hilbert space has a tensor product structure.

To complete the general discussion we must also show how to couple different encoded qubits through a nontrivial (entangling) gate. For the $X X Z$ model this turns out to be even simpler than implementing singleencoded-qubit operations. For, turning on the coupling $J_{2 m, 2 m+1}^{z}$ between pairs of spins belonging to two neighboring encoded qubits immediately implements the encoded $-T_{m}^{z} T_{m+1}^{z}\left(R_{m}^{z} R_{m+1}^{z}\right)$ Hamiltonian on the axially symmetric (antisymmetric) qubits. To see this, note that in the axially symmetric case, e.g., $\left|0_{L}\right\rangle_{1}\left|0_{L}\right\rangle_{2}=$ $|01\rangle_{12}|01\rangle_{34} \stackrel{\sigma_{2}^{z} \sigma_{3}^{z}}{\rightarrow}-|01\rangle_{12}|01\rangle_{34}=-\left|0_{L}\right\rangle_{1}\left|0_{L}\right\rangle_{2}$, and similarly for the other three combinations: $\left|0_{L}\right\rangle\left|1_{L}\right\rangle \rightarrow$ $\left|0_{L}\right\rangle\left|1_{L}\right\rangle,\left|1_{L}\right\rangle\left|0_{L}\right\rangle \rightarrow\left|1_{L}\right\rangle\left|0_{L}\right\rangle,\left|1_{L}\right\rangle\left|1_{L}\right\rangle \rightarrow-\left|1_{L}\right\rangle\left|1_{L}\right\rangle$ so that in all $\sigma_{2}^{z} \sigma_{3}^{z}$ indeed acts as $-T_{1}^{z} T_{2}^{z}$. Since as is well known [19], the controlled-phase (CPHASE) gate is directly obtainable by turning on the Hamiltonian $\sigma^{z} \otimes \sigma^{z}$ between physical qubits, in our case turning on $J_{2 m, 2 m+1}^{z}$ yields a CPHASE gate between encoded qubits. We consider the $X Y$ model below, since it requires the introduction of the selective recoupling method. For the $X X Z$ model, this encoded-CPHASE together with the single-encoded-qubit operations suffices to perform encoded universal quantum computation 20]. 
Recoupling and Encoding Recoupling. - Recall that, as discussed above, in each instance of $H_{\text {ex }}$ (Heisenberg, $X Y, X X Z)$ one typically has control over only one type of parameter out of the set $\left\{J_{i j}^{\alpha}, \varepsilon_{i}\right\}$. In our treatment above we made liberal use of all parameters, and it is now time to relax this assumption. To show this we now demonstrate how selective recoupling, applied to our encoded qubits, provides the requisite flexibility. Let us first briefly recall the basic idea of selective recoupling [16] through a simple NMR example. In a 2-spin molecule in NMR, the internal Hamiltonian is $H_{\mathrm{NMR}}=\sum_{i=1}^{2} \varepsilon_{i} \sigma_{i}^{z}+J_{12}^{z} \sigma_{1}^{z} \sigma_{2}^{z}$ with uncontrollable parameters $\varepsilon_{i}, J_{12}^{z}$. However, control over $\varepsilon_{i}$ is needed to implement $z$-axis rotations, while control over $J_{12}^{z}$ is needed to implement a CPHASE. This is done by pulsing an external magnetic field along the $x$-axis. Let $A$ and $B$ be anticommuting hermitian operators where $A^{2}=I$ ( $I$ is the identity matrix). Then the operation of "conjugating by $A "$,

$$
\begin{aligned}
C_{A} \circ \exp (i B) & \equiv \exp (-i A \pi / 2) \exp (i B) \exp (i A \pi / 2) \\
& =\exp (-i B)
\end{aligned}
$$

causes $B$ 's sign to be flipped. Thus: $\exp \left(-i H_{\mathrm{NMR}} \tau\right)\left[C_{\sigma_{1}^{x}} \circ \exp \left(-i H_{\mathrm{NMR}} \tau\right)\right]=\exp \left[-2 i \tau \varepsilon_{2} \sigma_{2}^{z}\right]$, which implements a rotation through an angle $\theta=2 \tau \varepsilon_{2}$ about the $z$-axis of the second spin. Notably, the Ising coupling term $\sigma_{1}^{z} \sigma_{2}^{z}$ has been eliminated. A similar calculation reveals that: $\exp \left(-i H_{\mathrm{NMR}} \tau\right)\left[C_{\sigma_{2}^{x}} \circ C_{\sigma_{1}^{x}} \circ \exp \left(-i H_{\mathrm{NMR}} \tau\right)\right]=$ $\exp \left[2 i \tau J_{12}^{z} \sigma_{1}^{z} \sigma_{2}^{z}\right]$, i.e., the selective implementation of the Ising coupling term through an angle $\tau J_{12}^{z}$. Notice that to achieve this effect all that was needed was control over the parameters turning on/off the $\sigma_{i}^{x}$ terms. Physically, the reason that $H_{\mathrm{NMR}}$ was neglected during the $\pi / 2$ rotations is that typically in NMR the $\sigma_{i}^{x}$ terms can be made much larger than $H_{\mathrm{NMR}}$. Selective recoupling methods can be extended to deal with any number of spins coupled through an NMR-type Hamiltonian, and efficient methods using Hadamard matrices have been developed for both homonuclear [21] and heteronuclear systems 22,23].

Consider now an $X X Z$-type Hamiltonian where the $J_{m}^{+}$parameters are controllable but $\epsilon_{m}^{-}$and $J_{m}^{z}$ are fixed. As argued above, this is a model of the $X Y$-examples of QC proposals 11 14 that takes certain symmetrybreaking mechanisms into account. We can now map the selective recoupling method directly onto our problem. For simplicity let us consider just the axially symmetric case. Then we can rewrite $H=H_{0}+H_{\mathrm{ex}}$ as: $H_{\mathrm{AX}}=\sum_{m=1}^{N / 2} \epsilon_{m}^{-} T_{m}^{z}-J_{m}^{z} T_{m}^{z} T_{m+1}^{z}+J_{m}^{+} T_{m}^{x}$, where we have omitted a constant term. The important point is now that $T_{m}^{x}$ and $T_{m}^{z}$ satisfy the properties required of $A$ and $B$ above, on the code subspace. In fact, the structure of $H_{\mathrm{Ax}}$ is exactly analogous to that of $H_{\mathrm{NMR}}$, the only difference being that the $T$ operators act on encoded qubits as opposed to directly on physical spins. Hence the argument that held for $H_{\mathrm{NMR}}$ holds here as well: By using recoupling through "conjugation by $T_{m}^{x}$ " we can selectively turn on and off the single-encoded-qubit rotation $T_{m}^{z}$ and the encoded-Ising interaction $T_{m}^{z} T_{m+1}^{z}$. This example of "encoded selective recoupling" establishes that encoded universal computation in the $X X Z$ model can be done using control over the $J_{m}^{+}$parameters alone.

Next, consider the $X Y$ model, i.e., the idealized version of the proposals in [11 14, with controllable $J_{i j}^{+}$, but fixed $\varepsilon_{i}$. We still use the encoding $\left|0_{L}\right\rangle=|\uparrow \downarrow\rangle$, $\left|1_{L}\right\rangle=|\downarrow \uparrow\rangle$. To implement encoded single-qubit operations, we can use the same encoded recoupling method as for the $X X Z$ model. As for encoded two-qubit operations, we now no longer have the $\sigma_{i}^{z} \sigma_{j}^{z}$ terms. Since the $X Y$ model with nearest-neighbor interactions can be shown not to be universal [17], we turn on also nextnearest neighbor $J_{i j}^{+}$terms (these can still be nearestneighbor in a $2 \mathrm{D}$ hexagonal geometry). First note that $C_{T_{12}^{x}} \circ T_{23}^{x}=i \sigma_{1}^{z} \sigma_{2}^{z} T_{13}^{x}$. Now assume we can control $J_{13}^{+}$; then, using conjugation by $\pi / 4: C_{\frac{1}{2} T_{13}^{x}} \circ\left(C_{T_{12}^{x}} \circ T_{23}^{x}\right)=$ $\sigma_{2}^{z}\left(\sigma_{3}^{z}-\sigma_{1}^{z}\right) / 2$. Since $\sigma_{1}^{z} \sigma_{2}^{z}$ is constant on the code subspace it can be ignored. On the other hand, $\sigma_{2}^{z} \sigma_{3}^{z}$ again acts as $-T_{1}^{z} T_{2}^{z}$, i.e., as an encoded $\sigma^{z} \otimes \sigma^{z}$. This establishes universal encoded computation in the $X Y$ model.

Cost. - Let us now count how many elementary steps are needed to implement the various quantum computing primitives in the $X X Z$ model. We define such a step as a single pulse whereby a single $J_{i j}^{\alpha}$ is switched on and then off. We will only assume the least demanding architecture of nearest neighbor interactions and a 1D layout of spins. Improvements are certainly possible with nextnearest neighbor interactions and/or a 2D geometry. For single-encoded-qubit operations, it takes one step to turn on a rotation about the encoded $x$-axis (under the standard assumption that we can make $\left.\left|J_{m}^{+}\right| \gg\left|\epsilon_{m}^{-}\right|,\left|J_{m}^{z}\right|\right)$, while it takes 4 steps to implement a rotation about the encoded $z$-axis (turn on $T_{m}^{x}$ for $\pi / 2$, free evolution under $\epsilon_{m}^{-} T_{m}^{z}-J_{m}^{z} T_{m}^{z} T_{m+1}^{z}$, repeat with $T_{m}^{x}$ for $\left.-\pi / 2\right)$. Therefore using the standard Euler angle construction it takes at most 6 steps to implement any single-encoded-qubit rotation. The encoded CPHASE operation is similar: If we assume that $T_{m}^{x}$ and $T_{m+1}^{x}$ can be turned on in parallel then the same count of 4 steps as for encoded $z$ axis rotations applies; otherwise we need to add 2 more operations, for a total of 6 steps. In the $X Y$ case the implementation of CPHASE given above takes 5 steps.

Encoded recoupling for the Heisenberg case.- Elimination of single-qubit operations in the isotropic exchange case was first shown in [3], and further developed in [1 6]. With a fully degenerate $H_{0}$ this required encoding one qubit into three. We now show how to simplify this encoding, assuming a non-degenerate $H_{0}$. Our code is the same as for the axially symmetric case above, and the same as that used in [8]9], but our method is less strin- 
gent. Ref. [8] used spin-resonance techniques, with the rather demanding requirement that the spin-spin interaction strength be modulated at high frequency. Ref. [9] showed how universal computation can be be performed by varying the strength of the exchange coupling using non-oscillatory pulses. This scheme is very much in the spirit of our solution, but it has the problem of undesired spin-rotations taking place while the interaction is off. We solve this problem here using the selective recoupling method. Let us write the total Hamiltonian as $H=H_{0}+H_{\text {Heis }}$, where $H_{\text {Heis }}=\sum_{i<j} J_{i j}\left(T_{i j}^{x}+\frac{1}{2} \sigma_{i}^{z} \sigma_{j}^{z}\right)$ with $\frac{1}{2} J_{i j}^{+}=J_{i j}^{x}=J_{i j}^{y}=J_{i j}^{z}=J_{i j}$. The exchange coupling parameters $J_{i j}$ are assumed to be controllable, while $H_{0}$ is not (except by application of a global magnetic field). Therefore, similar to the anisotropic case, when we turn on $J_{m} \equiv J_{2 m-1,2 m}$ the Hamiltonian can be written as $H_{\text {Heis }}=\sum_{m=1}^{N / 2}\left(\epsilon_{m}^{-} T_{m}^{z}+J_{m} T_{m}^{x}\right)+\Delta$, where $\Delta=\sum_{m=1}^{N / 2}\left(\epsilon_{m}^{+} R_{m}^{z}+\frac{J_{m}}{2} \sigma_{2 m-1}^{z} \sigma_{2 m}^{z}\right)$ acts trivially on the code space and hence can be omitted. By recoupling using $T_{m}^{x}$ we can selectively turn on and off the singleencoded-qubit rotation $T_{m}^{z}$, as above, thus generating the encoded- $S U(2)$ group only on the desired encodedqubit. Next, let us show how to selectively turn on a two-qubit Hamiltonian such as $T_{1}^{z} T_{2}^{z}=\sigma_{2}^{z} \sigma_{3}^{z}$. If we directly turn on $h_{23}=J_{23} \sum_{\alpha=x, y, z} \sigma_{2}^{\alpha} \sigma_{3}^{\alpha}$, the encoded space will leak. Recoupling can extract the $\sigma_{2}^{z} \sigma_{3}^{z}$ term as follows. First, note that $C_{T_{1}^{x}} \circ e^{-i \pi T_{1}^{z} / 2}=e^{i \pi T_{1}^{z} / 2}$, and $C_{T_{1}^{z}} \circ h_{23}=J_{23}\left(-\sigma_{2}^{x} \sigma_{3}^{x}-\sigma_{2}^{y} \sigma_{3}^{y}+\sigma_{2}^{z} \sigma_{3}^{z}\right)$. Hence $e^{-i h_{23} t / 2} C_{T_{1}^{z}} \circ e^{-i h_{23} t / 2}=e^{-i J_{23} \sigma_{2}^{z} \sigma_{3}^{z} t}$, so that $T_{1}^{z} T_{2}^{z}$ may be implemented selectively using 6 steps, which completes the requirements for universal computation. It is interesting to contrast these results with the 19 steps required in serial mode for the analogous operation in the isotropic case, assuming fully degenerate $H_{0}$ (7 steps are required in parallel mode in 2D) [6]. As a final comment, note that the code we used here is a decoherence-free subspace (DFS) and thus offers automatic protection against collective dephasing errors [П], as recently demonstrated in an ion trap experiment [24]. When other errors are present one may use the method of concatenating DFSs with quantum error correcting codes [25], at the price of introducing greater qubit overhead, or use a combination of recoupling and decoupling techniques 26 .

Conclusions. - The requirement of performing both single- and two-qubit operations in one quantum computing device often leads to severe technical constraints and difficulties. We have shown here that selective recoupling, as applied to encoded qubits, is a very general method to overcome these problems. It allows all quantum logic operations to be performed by turning on/off pairwise exchange interactions. The trade-off is modest: a qubit is encoded into the state of two neighboring spins, and universal quantum logic gates require only 4-6 interactions to be turned on/off in a simple 1D geometry with nearest neighbor coupling. We believe that this alternative to the hard requirements of quantum computing with single-qubit gates may substantially simplify the design of quantum computers.

Acknowledgements. - DAL acknowledges support from PREA, PRO, the Connaught Fund, and AFOSR (F49620-01-1-0468). We thank Profs. V. Privman, A. Imamoḡlu, and B.E. Kane for useful correspondence.

[1] D. Loss and D.P. DiVincenzo, Phys. Rev. A 57, 120 (1998); G. Burkard et al., Phys. Rev. B 59, 2070 (1999); X. Hu and S. Das Sarma, Phys. Rev. A 61, 062301 (2000).

[2] B.E. Kane, Nature 393, 133 (1998); R. Vrijen et al., Phys. Rev. A 62, 012306 (2000).

[3] D. Bacon et al., Phys. Rev. Lett. 85, 1758 (2000).

[4] J. Kempe et al., Phys. Rev. A 63, 042307 (2001).

[5] L. Viola et al., Phys. Rev. Lett. 85, 3520 (2000).

[6] D.P. DiVincenzo et al., Nature 408, 339 (2000).

[7] D. Bacon et al., eprint quant-ph/0102140.

[8] J. Levy, eprint quant-ph/0101057.

[9] S.C. Benjamin, Phys. Rev. A 64, 054303 (2001).

[10] K.V. Kavokin, Phys. Rev. B 64, 075305 (2001).

[11] D. Mozyrsky et al., Phys. Rev. Lett. 86, 5112 (2001).

[12] A. Imamoḡlu et al., Phys. Rev. Lett. 83, 4204 (1999).

[13] L. Quiroga and N.F. Johnson, Phys. Rev. Lett. 83, 2270 (1999).

[14] S.-B. Zheng and G.-C Guo, Phys. Rev. Lett. 85, 2392 (2000), and references therein.

[15] P.M. Platzman and M.I. Dykman, Science 284, 1967 (1999).

[16] J.S. Waugh et al., Phys. Rev. Lett. 5, 180 (1968); C. Slichter, Principles of Magnetic Resonance (Springer, Berlin, 1996).

[17] L.-A. Wu and D.A. Lidar, eprint quant-ph/0103039 4; quant-ph/0109078.

[18] When spin-orbit interaction is taken into account this must be modified to include cross terms 10. We will analyze this perturbation in a forthcoming publication.

[19] M.A. Nielsen and I.L. Chuang, Quantum Computation and Quantum Information (Cambridge University Press, Cambridge, UK, 2000).

[20] A. Barenco et al., Phys. Rev. A 52, 3457 (1995).

[21] N. Linden et al., Chem. Phys. Lett. 311, 321 (1999).

[22] D.W. Leung et al., Phys. Rev. A 61, 042310 (2000).

[23] J.A. Jones and E. Knill, J. Magn. Reson. 141, 322 (1999).

[24] D. Kielpinski et al., Science 291, 1013 (2001).

[25] D.A. Lidar et al., Phys. Rev. Lett. 82, 4556 (1999).

[26] M.S. Byrd and D.A. Lidar, quant-ph/0112054. 


\begin{tabular}{|c|c|c|c|}
\hline System & Two-qubit Hamiltonian & $H_{0}=\sum_{i} \frac{1}{2} \varepsilon_{i} \sigma_{i}^{z}$ & External $f_{i}^{x, y}$ \\
\hline Spin-coupled quantum dots & Heisenberg, controllable & fixed & hard \\
\hline Donor atom nuclear/electron spins 2 & Heisenberg, controllable & fixed & hard \\
\hline Quantum Hall 11 & $X Y$, controllable & fixed & hard \\
\hline Quantum dots/atoms in cavities 12, 14 & $X Y$, controllable & controllable & easy, requires additional lasers \\
\hline Exciton-coupled quantum dots $\mid 13$ & $X Y$, controllable & fixed & hard \\
\hline Electrons on helium 15 & $X X Z$, only $J_{i j}^{+}$controllable & controllable & easy but slow and hard to tune \\
\hline
\end{tabular}

TABLE I. Comparison of some QC proposals in terms of difficulty of implementing two-qubit $\left(J_{i j}^{\alpha}\right)$, internal $\left(\epsilon_{i}\right)$, and external single-qubit operations $\left(f_{i}^{x, y}\right) . H_{0}=$ fixed means that it is hard to independently change each $\varepsilon_{i}$. 